\title{
Effective dose and image quality evaluations of an automatic CT tube current modulation system with an anthropomorphic phantom
}

\author{
O. Rampado a,*, F. Marchisio ${ }^{\text {b }}$, A. Izzo ${ }^{\text {a }}$, E. Garelli ${ }^{a}$, C.C. Bianchi ${ }^{b}$, G. Gandini ${ }^{b}$, R. Ropolo ${ }^{\mathrm{a}}$ \\ a Medical Physics Department, San Giovanni Battista Hospital of Turin, Corso Bramante 88, 10126 Torino, Italy \\ b Radiodiagnostic Section, Medical-Surgical Sciences Department, University of Turin, Via Genova 3, 10126 Torino, Italy
}

\section{A R T I C L E I N F O}

\section{Article history:}

Received 1 April 2008

Received in revised form 4 June 2008

Accepted 25 June 2008

\section{Keywords:}

Computed tomography

Patient dose

Image quality

Automatic exposure

\begin{abstract}
A B S T R A C T
The purpose of this study was to evaluate the consequences of different choices of acquisition parameters on the actual image noise and on the patient dose with an automatic tube current modulation system. The CT investigated was a GE Lightspeed 16-slice and an anthropomorphic phantom was used to simulate the patient. Several acquisitions were made varying noise index (NI), kilovoltage and pitch values. Tube current values were compared for the different acquisitions. Patient dose was evaluated in terms of volumetric computed tomography dose index $\left(\mathrm{CTDI}_{\mathrm{vol}}\right)$ and also as effective dose. The noise actually present in the images was analyzed by a region of interest analysis considering representatively phantom sections in the regions of the shoulders, of the lungs and of the abdomen. The obtained results generally evidenced a good agreement between the noise index and the measured noise for the abdomen sections, whereas for the shoulders and the lungs sections the measured noise was respectively greater and lower of the NI. Varying the $\mathrm{kV}$ the automatic current modulation system provided images with a substantially constancy of the actual noise and of the patient dose. An increase of the pitch generally decreased the patient dose, whereas the noise was slightly greater for the lowest pitch and almost constant for the other pitch values. This study outlines some important relationships between an automatic tube current modulation system and other CT acquisition parameters, providing useful informations for the choice requested by radiologists in the task of optimization of the $\mathrm{CT}$ acquisition protocols. Unless there are other considerations in place, pixel pitches below 1.375 should be avoided, and $\mathrm{kVp}$ settings can be changed with no real impact on dose or image noise.
\end{abstract}

(c) 2008 Elsevier Ireland Ltd. All rights reserved.

\section{Introduction}

The rapid technological evolution of the computed tomography (CT) equipments challenges the radiologists community to continuously optimize acquisition protocols, with the goal of obtaining diagnostic images with the lowest achievable patient dose. In the last years, one of the most important improvements was the implementation of automatic tube current modulation systems [1,2]. With this system it is possible to set in the acquisition protocol the desired level of image quality. Based on this parameter the tube current is automatically defined in order to obtain a constant level of noise over all the scanned regions. As a consequence one of the most important parameter is now the value of the "desired noise" that is specified in different ways for the different CT manufac-

\footnotetext{
* Corresponding author at: S.C. Fisica Sanitaria, A.S.O. San Giovanni Battista di Torino, Corso Bramante 88, 10126 Torino, Italy. Tel.: +39 0116335373 ; fax: +390116634547.

E-mail address: orampado@molinette.piemonte.it (O. Rampado).
}

turers. Depending on the software, the modulation system varies the current along the $Z$-axis direction or in the $X-Y$ plane keeping into account the different thickness and attenuation property of the patient in the antero-posterior and lateral directions. Several studies on both phantoms and patients have demonstrated the efficacy of these systems in reducing patient dose [3-8].

However, few published works investigated the behaviour of these automatic current modulation systems when other acquisition parameters are changed. With a current modulation system the dose and image quality dependences on other acquisition parameters, like the kilovoltage or the pitch value, are different from the case of constant current and investigation of different acquisition protocols should keep into account this fact. Goo and Suh [9] explored the consequence of using different $\mathrm{kV}$ values and found differences in terms of volumetric computed tomography dose index $\left(\mathrm{CTDI}_{\mathrm{vol}}\right)$ and $\mathrm{mAs}$ between the unmodulated and the modulated scans. Analysis of how the beam width and the pitch factor selection affect the efficacy of the tube current modulation has been done by Papadakis et al. [10]. However, as outlined in Section 4 of these studies, the results are strictly valid 
for the CT used and the associated tube current modulation systems. The different manufacturers adopted different solutions to modulate the tube current and, as a consequence, it is important to study how the different acquisition parameters influence the dose reduction achievable by tube current modulation for the system in use.

The purpose of this study was to evaluate the effective dose and the actual noise present in the CT images of an anthropomorphic phantom when an automatic tube current modulation system is used with different values of the other acquisition parameters.

\section{Materials and methods}

CT scans were performed with a multislice CT (Lightspeed 16Pro, GE, Milwakee, WI, USA). The "AutomA" option was set for all the scan in order to have the longitudinal tube current modulation. This modulation adjusts tube current to maintain an operator-defined noise level in the reconstructed images that is predominately independent of patient size and anatomy. The parameter used to specify the desired image quality was the noise index (NI). The NI value is specified by the vendor as approximately equal to the SD in the central region of the image when a uniform phantom is scanned and reconstructed using the standard reconstruction algorithm. A detailed analysis of the theoretical relationship between NI and radiation dose has been reported in [11].

With this "AutomA" tool it is also possible to set the minimum and the maximum $\mathrm{mA}$ values used during the scan. In the present study, in order to observe the current modulation trend without limitations, these parameters were always set to the absolute minimum and maximum current values of the scanner.

An anthropomorphic phantom (3D Torso Phantom, CIRS, Norfolk, VA, USA) was used to simulate a typical adult patient. This phantom is made of radiologically tissue-equivalent material, and its internal structure includes artificial skeleton, lung, and soft tissues (muscle, heart, liver, pancreas, kidneys) formulated to simulate the physical density and linear attenuation of actual tissues to within $2 \%$ in the diagnostic energy range.

The examination protocol parameters used to first scan the phantom were as follow: $120 \mathrm{kV}$, NI 21 , rotation time $0.5 \mathrm{~s}$, slice thickness $1.25 \mathrm{~mm}$, beam collimation $20 \mathrm{~mm}(16 \times 1.25)$, pitch 1.375 , FOV $36 \mathrm{~cm}$. Other scans were next performed varying the $\mathrm{NI}$ in the range 15-27 while maintaining constant the other parameters. In order to investigate the current modulation with different $\mathrm{kV}$, four scans were subsequently performed at a 21-NI level with a tube voltage of $80,100,120$ and $140 \mathrm{kV}$. A similar approach was used to study the pitch dependence with scans with pitch 0.563 (9/16), 0.938 (15/16), 1.375 (22/16) and 1.75 (28/16).

The tube current values for all the acquired scans were recorded after extracting the information from the DICOM header of the stored images by means of a self-developed software. These value were then multiplied by the rotation time used to obtain the mAs values. The $\mathrm{CTDI}_{\mathrm{vol}}$ provided by the equipment user interface was recorded for every exposure condition. The accuracy of these provided values was verified by comparison with values measured during the routinely quality controls, finding a maximum difference of $5 \%$.

The patient dose was also evaluated in terms of effective dose. The software IMPACT CT PATIENT DOSE CALCULATOR [12] was used, after a modification of the calculation procedure to consider the actual distribution of organ dose consequent to the current modulation. We developed a MS Excel macro that considers the $\mathrm{mA}$ current values for every slab of thickness $1 \mathrm{~cm}$ of the scanned volume, it calculates the relative effective dose contribution and adds all the contributions to determine the total effective dose.
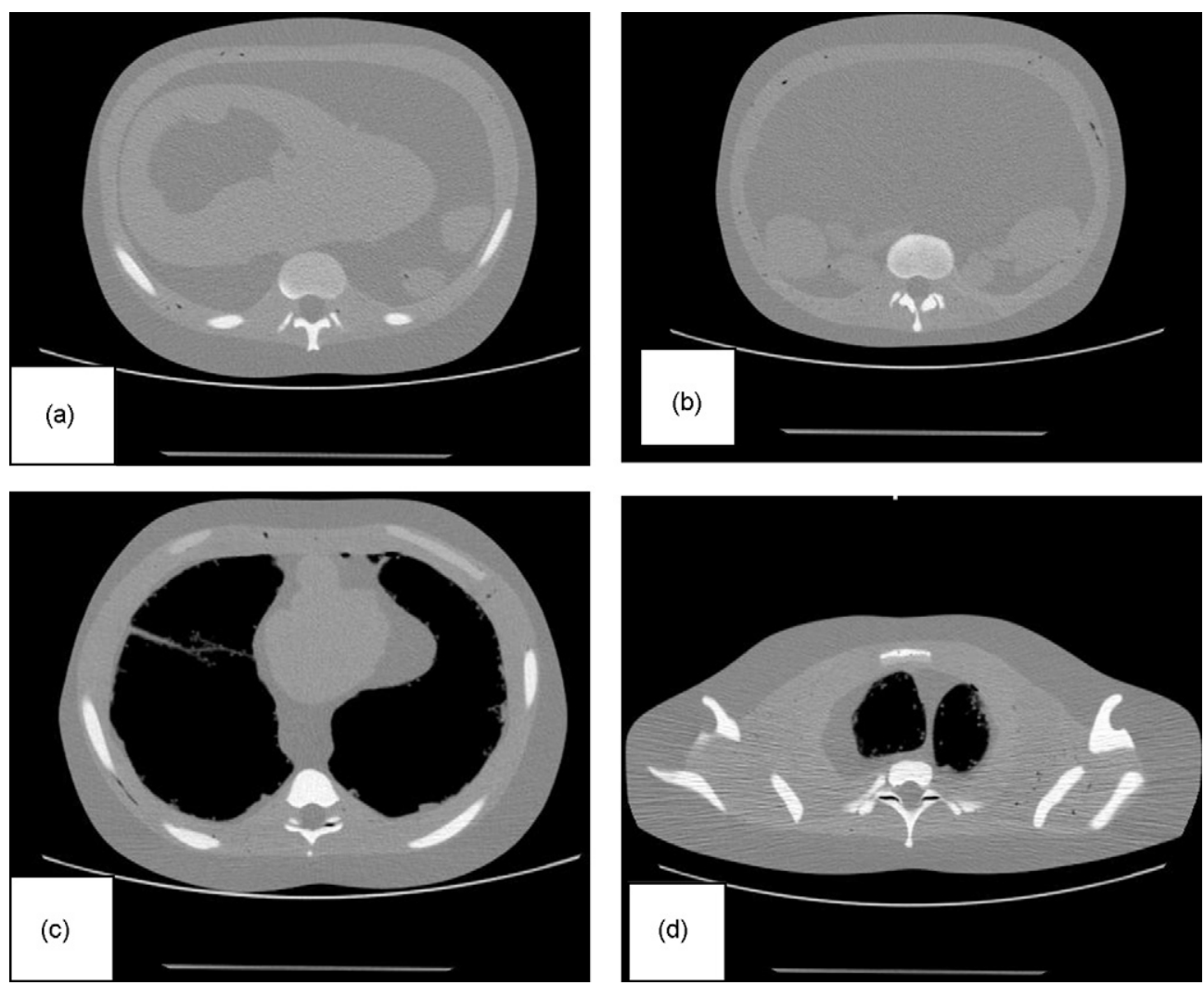

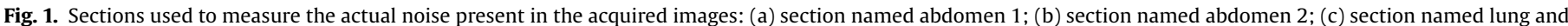
(d) section named shoulders. 
In order to compare the actual noise present in all the acquired series with the chosen NI, four sections were considered: two in the abdominal region (at $Z$ positions respectively of a minimum and a maximum of tube current), one in the thorax region (at $Z$ position of the minimum current) and one in the shoulder region (at $Z$ position of the maximum of current). The position of the four sections is indicated in Fig. 1. For each of these sections some regions of interest were considered in the soft tissue portions of the image and standard deviation values in Hounsfield Units were measured and recorded to quantify the actual noise.

Further measurements were performed on a similar CT equipment (Lightspeed 16, GE, Milwakee, WI, USA). The main difference between the GE Lightspeed 16 and the GE Lightspeed Pro16 is that the Lightspeed Pro16 has a different X-ray tube, generator and gantry to enable higher $\mathrm{mA}$ techniques and faster scan times. Both models have the same detector layout consisting of sixteen $0.63 \mathrm{~mm}$ detector banks and eight $1.25 \mathrm{~mm}$ detector banks. Also the software "AutomA" for the $\mathrm{mA}$ modulation is the same for these two CT equipments. With the aim of verify if the relative dependence of noise and dose from the acquisition parameters were the same for these two CT equipments, part of the measurements previously described were also performed on the Lighstpeed 16 and the obtained results were compared.

\section{Results}

Part of the figures presented in this study shows the tube current time product ( $\mathrm{mAs}$ ) versus the longitudinal coordinate $Z$. The rotational time is obviously constant, so the trend shown corresponds to the tube current trend. We choice to represent the mAs values and not the $\mathrm{mA}$ values in order to facilitate comparisons between acquisition performed with a different choice of the rotational time.

Fig. 2(a) shows the mAs trend along the longitudinal axis for the standard protocol. The highest tube current values were observed for the shoulders and the upper abdomen, whereas lower current values were found for the lungs region. The plot in Fig. 2(b) represents an example of $\mathrm{mAs}$ trend for an actual patient and it is possible to see that relative maximums and minimums of current were located in the positions similar to those observed for the phantom.

Fig. 3 shows the mAs values obtained for the scanned region for different NI settings. The mAs trend is the same for all the NI considered, with absolute values depending on the well known relationship between the noise and the tube current. The influence of the NI settings on the patient dose is also shown in Fig. 4, where it is possible to see that the average mAs value, the CTDI and the effective dose are all proportional to the inverse of the second power of the noise index.

Fig. 5 shows the actual noise values measured in the phantom. For the considered abdomen sections, minimum differences $(<6 \%)$ were observed between the measured noise and the NI chosen. The actual noise was greater of the NI chosen for the shoulders sections, where high density regions are comprised in the field of view and the $\mathrm{mA}$ modulation do not compensate the bone attenuation resulting in an increase of the noise. The differences between the measured noise and the actual noise for this section were in the range $35-60 \%$. Likewise, in the lung section the measured noise was different from the NI chosen, but with lower values as a consequence of the partial compensation of the scanned low density structures (differences of about -20\%). These differences are not in contrast with the NI definition that, as stated before, should reflect the standard deviation of a uniform phantom, so it is expected that for asymmetric anatomy the actual standard deviation could be different. However it is important to note that also in the lung and shoulder regions, there is a linear dependence of the actual noise
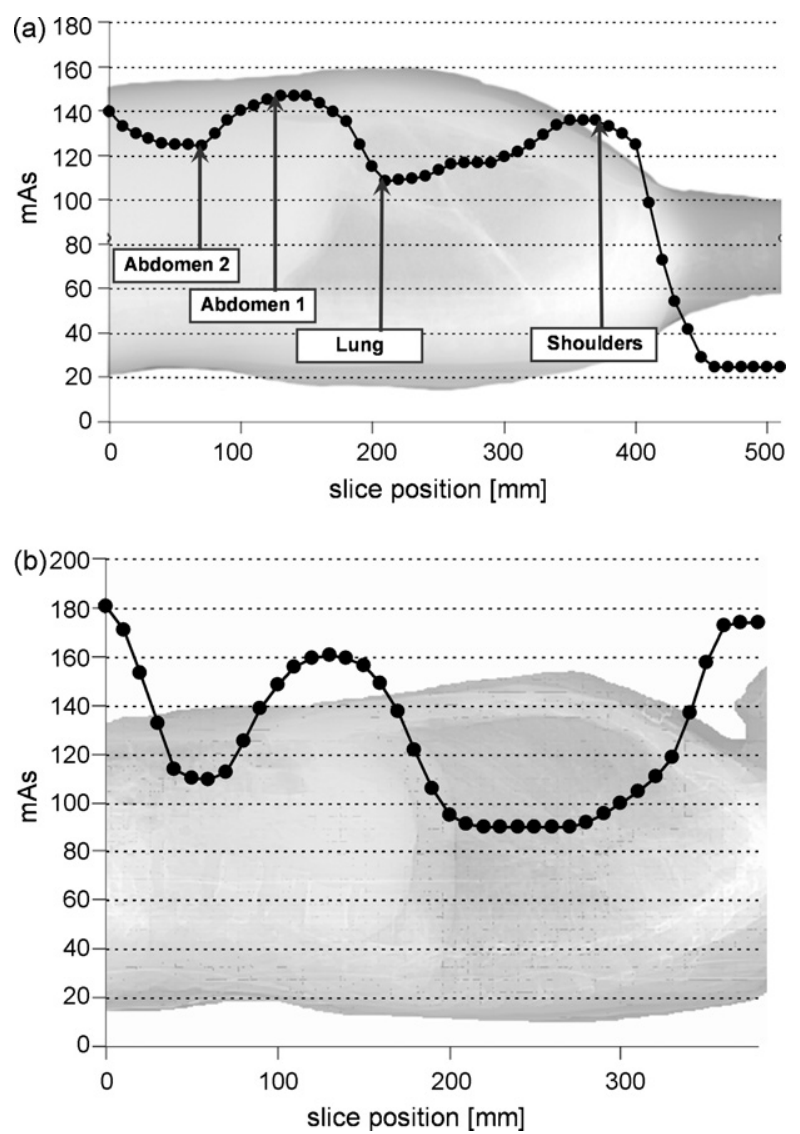

Fig. 2. (a) Tube current values along the longitudinal axis for the phantom acquisition, overlaid on the lateral scout view of the phantom used. The indication of the positions of the sections used to measure the actual noise is also shown. (b) Tube current values along the longitudinal axis for the routine acquisition protocol for a patient examination, overlaid on the lateral scout view of the patient.

versus the NI, so the percentage variation of the resulting standard deviation consequent to a NI variation can be calculated.

The mAs values observed for acquisitions performed with different tube voltage values and the same NI are showed in Fig. 6 and in Fig. 7(a). The standard tube voltage used for the abdomen-thorax acquisition protocol was $120 \mathrm{kV}$. Average mA values used for kilo-

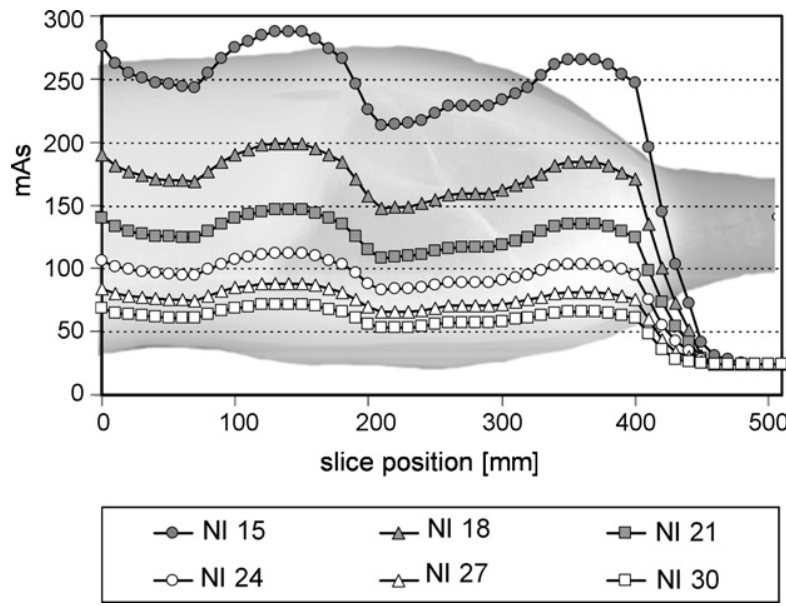

Fig. 3. mAs values along the longitudinal axis for different NI values, maintaining constant the other acquisition parameters $(120 \mathrm{kV}$, rotation time $0.5 \mathrm{~s}$, slice thickness $1.25 \mathrm{~mm}$, beam collimation $20 \mathrm{~mm}$, pitch 1.375 , FOV $36 \mathrm{~cm}$ ). 

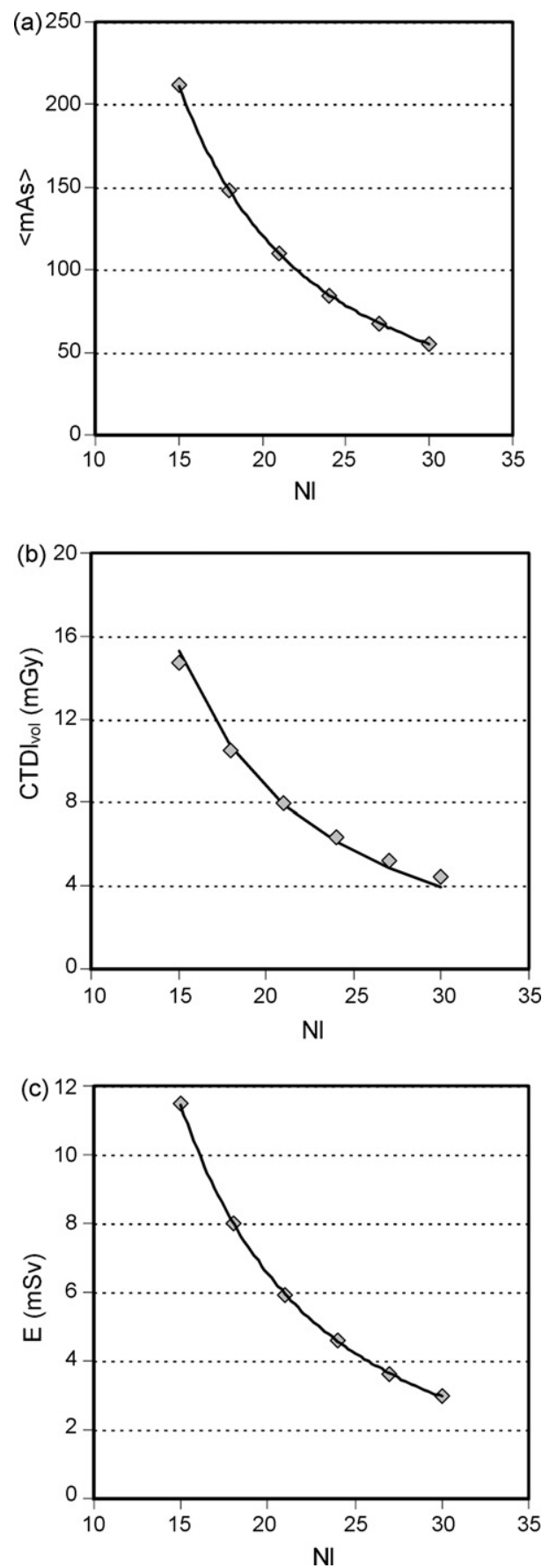

Fig. 4. Average $\mathrm{mAs}(\mathrm{a}), \mathrm{CTDI}_{\mathrm{vol}}$ (b) and effective dose values (c) calculated for the acquisitions performed with different NI values. The plotted lines represent curve fits of the type constant multiplied by the inverse of the second power of the noise index.

voltage values of 80 and $100 \mathrm{kV}$ were, respectively, $270 \%$ and $60 \%$ greater. Tube current values used for the different anatomical regions were not directly proportional for the different tube voltages and the modulation strategy kept into account the effect of the various density structures on the attenuation of the beam and the resulting noise. The $\mathrm{CTDI}_{\mathrm{vol}}$ values shown in Fig. 7(b) have an average value of $7.6 \mathrm{mGy}$, with a maximum of $8.5 \mathrm{mGy}$ (13\% greater) for $80 \mathrm{kV}$ and a minimum of $7.0 \mathrm{mGy}$ ( $7 \%$ lower) for the $140 \mathrm{kV}$. The graph of the effective dose versus $\mathrm{kV}$ shown in Fig. 7(c) outlines a substantial constancy of the patient dose for all the $\mathrm{kV}$ values with a constant NI chosen.

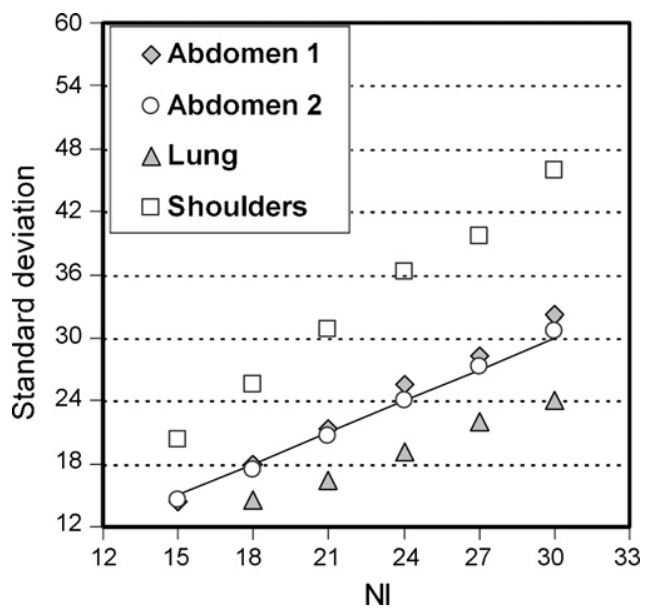

Fig. 5. Measured standard deviation for the four sections considered and for the different NI used. The continuous line represent the standard deviation equal to NI rect.

The actual noise values (Fig. 8) are comprised between $+2 \%$ and $-9 \%$ of the NI chosen for the two abdominal sections, whereas for the shoulder and lungs sections they are on average respectively $35 \%$ greater and 20\% lower. These results demonstrate that this automatic current modulation systems is able to correctly compensate for the different $\mathrm{kV}$ values selectable by the equipment user, providing images with noise values close to the NI chosen and maintaining a constant value of the patient dose.

The behaviour of the mAs selected by the automatic current modulation systems with different pitch values is shown in Fig. 9. The mAs trend for the scanned region was similar for the four pitches and the average current values increased when the pitch was incremented. The major difference was observed in the transition from pitch 0.938 to pitch 1.375 , with an increment of $40 \%$, whereas the tube current for the pitch 1.375 and 1.75 are more similar with differences lower than $12 \%$ (Fig. 10(a)). The patient dose trend for the different pitch values was different because it is proportional to the ratio between the tube current and the pitch value, so the greatest dose was observed for the pitch 0.563 (Fig. 10(b) and (c)).

Measured noise values (Fig. 11) revealed a greater noise for the minimum pitch of 0.563 . For the abdomen sections the measured noise of the images acquired with this pitch was about $17 \%$ greater

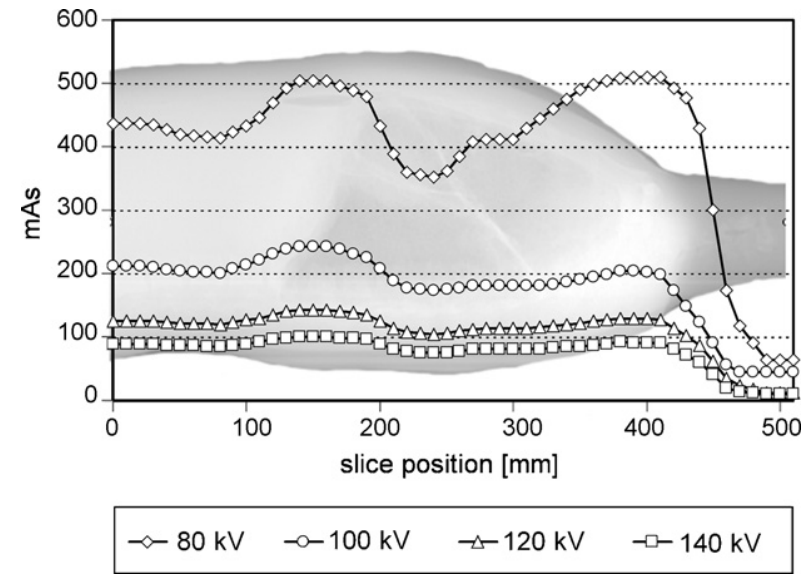

Fig. 6. $\mathrm{mAs}$ values along the longitudinal axis for different $\mathrm{kV}$ values, maintaining constant the other acquisition parameters (NI 21, rotation time $0.9 \mathrm{~s}$, slice thickness $1.25 \mathrm{~mm}$, beam collimation $20 \mathrm{~mm}$, pitch 1.375 , FOV $36 \mathrm{~cm}$ ) 

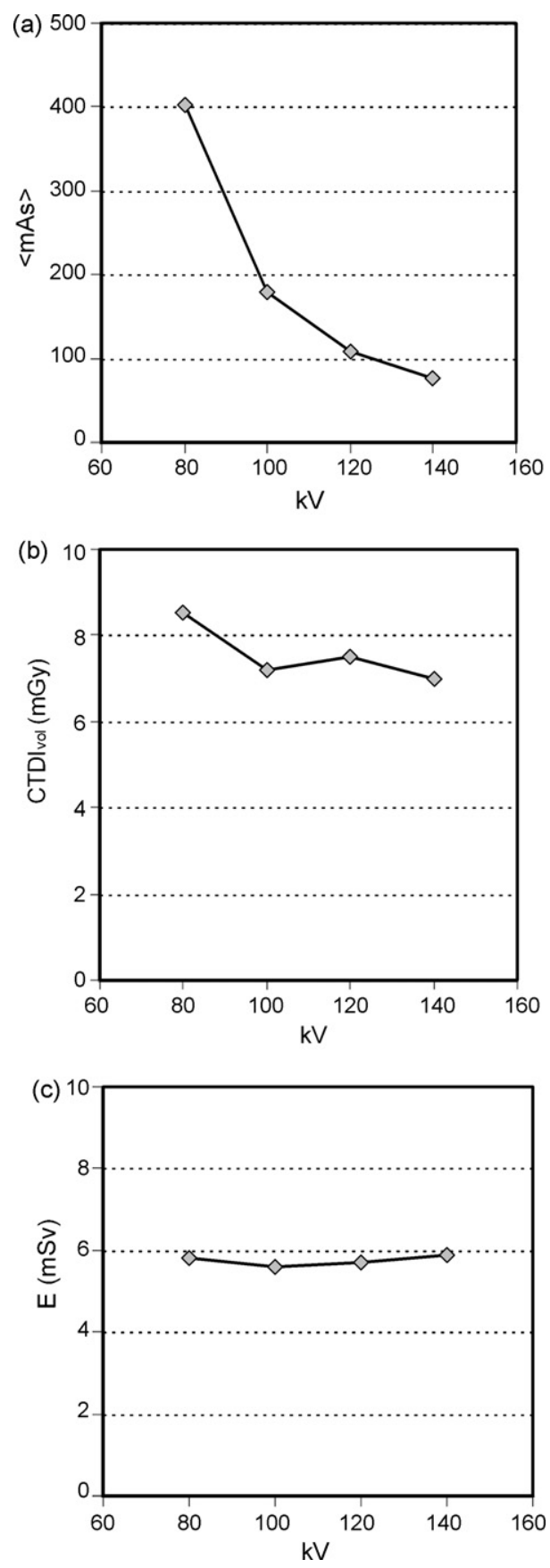

Fig. 7. Average current (a), $\mathrm{CTDI}_{\mathrm{vol}}$ (b) and effective dose values (c) calculated for the acquisitions performed with different $\mathrm{kV}$ values.

of the NI chosen, whereas the differences for the other pitch values were below $5 \%$. The noise was greater for the shoulder sections and lower for the lung section in a similar way of the results previously showed.

A comparison between the $\mathrm{CTDI}_{\mathrm{vol}}$ values obtained with the Lightspeed Pro16 CT and the Lightspeed 16 CT is shown in Fig. 12. Similar trends were observed and a maximum difference of $4.7 \%$ was obtained. The behaviour of dose and noise for different $\mathrm{kV}$ values was not investigated for the Lightspeed 16, because the equipment used in our radiological department is not calibrated for the use at 80 and $100 \mathrm{kV}$. Also the noise evaluated for the images of the Lightspeed 16 shows a trend similar to that previously described for the Lightspeed Pro16, both versus NI and versus the pitch. The

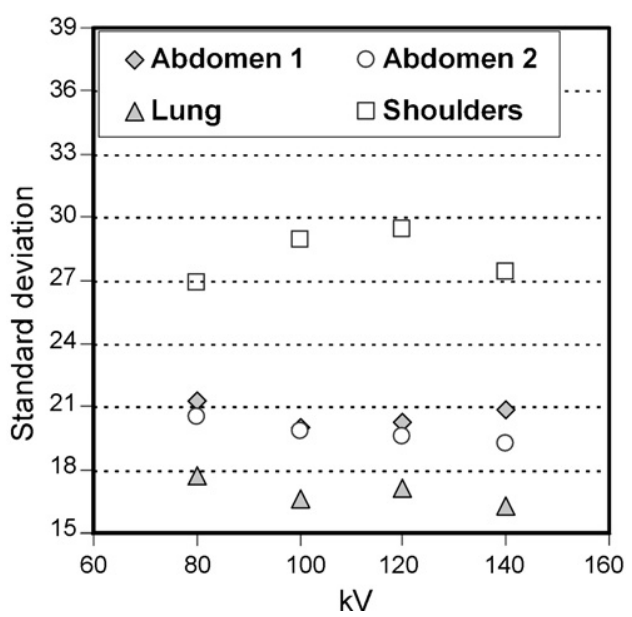

Fig. 8. Measured standard deviation for the four sections considered and for the different $\mathrm{kV}$ used.

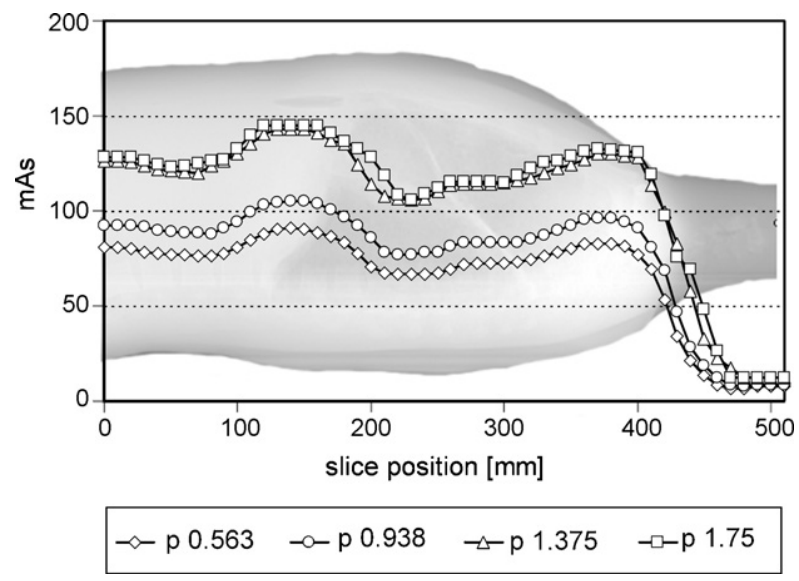

Fig. 9. $m$ As values along the longitudinal axis for different pitch values, maintaining constant the other acquisition parameters $(120 \mathrm{kV}$, NI 21 , rotation time $0.5 \mathrm{~s}$, slice thickness $1.25 \mathrm{~mm}$, beam collimation $20 \mathrm{~mm}$, FOV $36 \mathrm{~cm}$ ).

difference between the measured noise in the same images of the two equipments was below $7 \%$.

\section{Discussion}

Tube current modulation systems are an effective tool to reduce patient dose without compromising the image quality. However, also other acquisition parameters affects the dose and the image quality and the optimization of their values should be investigated. In this study, the consequences of different tube voltages and pitch selections on the patient dose and image quality were explored for a particular tube current modulation system.

Results showed that for an anthropomorphic adult phantom the current modulation system is able to provide approximately the same image noise and the same patient dose for the four tube voltage values available. In a previous study [9] the effect of $\mathrm{kV}$ on the behaviour of the tube current modulation was investigated, both on anthropomorphic and geometrical phantoms. The results are shown in terms of differences between the unmodulated and the modulated $\mathrm{CTDI}_{\mathrm{vol}}$ and in terms of noise, but also in this case it is possible to see that differences in $\mathrm{CTDI}_{\mathrm{vol}}$ between the three $\mathrm{kV}$ used were below $10 \%$ and the noise evaluated in air was comprised in a $\pm 3 \%$ range. In our study, patient dose was evaluated in terms of $\mathrm{CTDI}_{\mathrm{vol}}$ and effective dose. Comparison of these dose indexes 

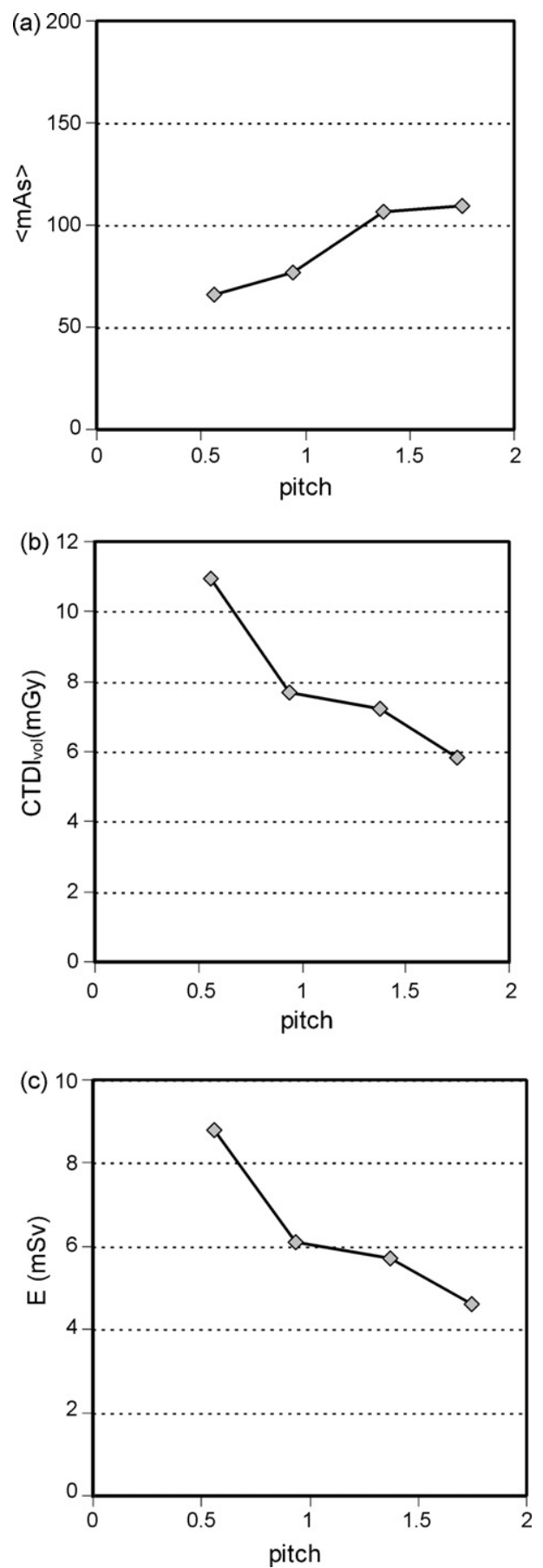

Fig. 10. Average current (a), $\mathrm{CTDI}_{\mathrm{vol}}$ (b) and effective dose values (c) calculated for the acquisitions performed with different pitch values.

for different tube voltages showed relative greater differences in terms of $\mathrm{CTDI}_{\mathrm{vol}}$, whereas the effective dose values were comprised in the range $5.7-5.9 \mathrm{mSv}$ (maximum difference $4 \%$ ). This different behaviour of the two dose indexes is a consequence of the effective dose calculation procedure and the dependence of the organ dose distribution on the beam spectrum. Effective dose is the best quantity to assess the radiological stochastic risk and we believe that in order to compare the patient dose with different tube voltages is better to consider the effective dose rather than the $\mathrm{CTDI}_{\mathrm{vol}}$.

On the base of these results it is possible to observe that other aspects related to the $\mathrm{kV}$ values, like the tissue contrasts or the

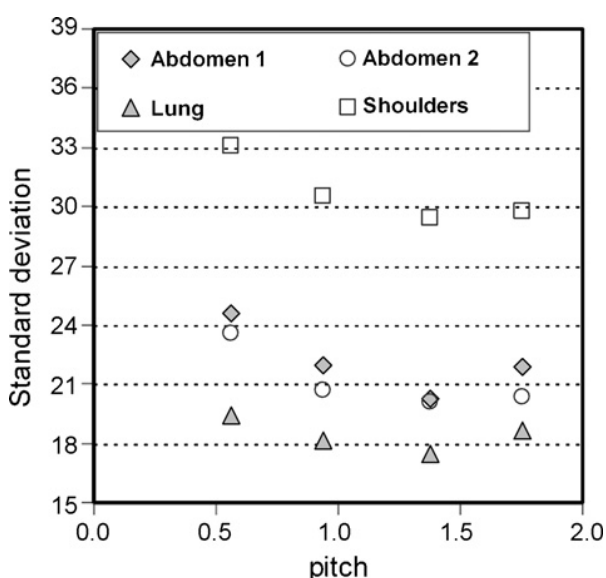

Fig. 11. Measured standard deviation for the four sections considered and for the different pitch used.

peculiar artefacts, can be investigated by the radiologists for specific exams or patient types considering that the constancy of the noise and of the patient dose is guaranteed by the automatic current modulation system. In recent years, the investigation of the optimum $\mathrm{kV}$ value for different kind of examinations is a topic of increasing interest and several studies have reported the possibility of reduction of patient dose or of the amount of contrast material using lower $\mathrm{kV}$ [13-16]. As an example, in a recent study the use of $80 \mathrm{kV}$ for thorax exams in adult patient was investigated [17] adjust-
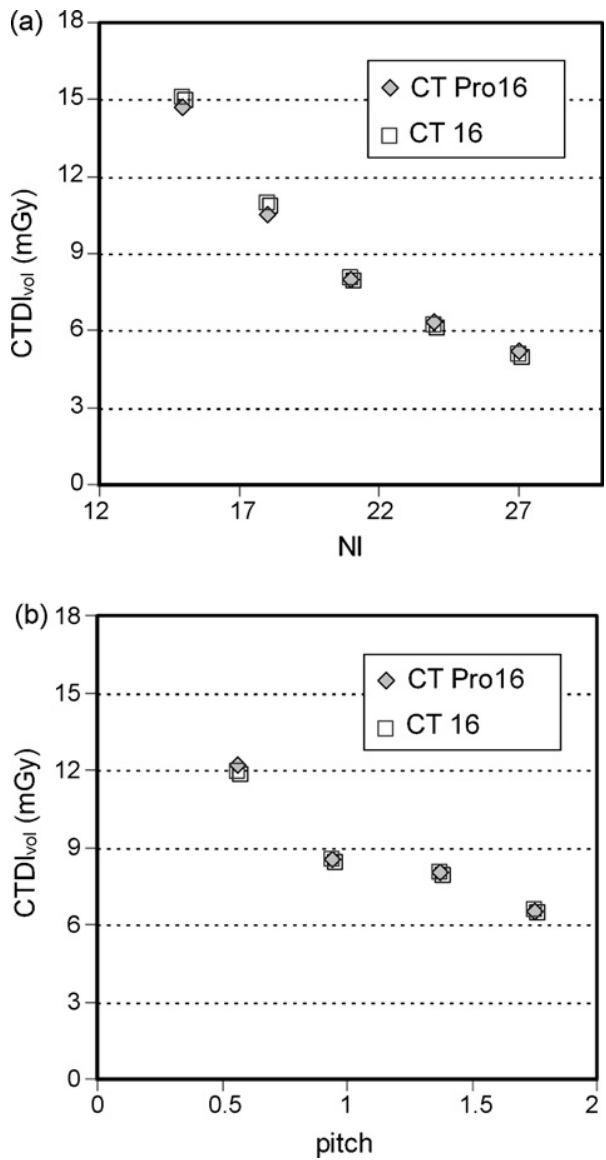

Fig. 12. Comparison between the $\mathrm{CTDI}_{\mathrm{vol}}$ values obtained for the acquisitions performed with different NI (a) and pitch values (b) for the CT GE Lightspeed 16 (CT 16) and the CT GE Lightspeed 16pro. 
ing manually the tube current values and a potential improvement in diagnostic quality images with reduction of contrast material and patient dose was found. With an automatic current modulation system like the one used in this work a similar study could be performed keeping dose and noise approximately constant in a first instance and elevating the NI maintaining the needed contrast in a second instance. We are currently investigating the consequences of the use of lower $\mathrm{kV}$ in the small peripheral vessel visualization and the preliminary results show improvements in image quality.

Both dose and image noise are dependent on the pitch selection, and the use of pitch values lower than 1.375 determines more noise and more patient dose, so its use should be justified by other needed improvements in image quality. These effects of the pitch and NI choice on the patient dose and effective image quality were probably correlated to the complex relationships between the image quality and pitch values for the multislice CT. As outlined in other works the noise is strongly dependent on the reconstruction algorithm and on the weighting function used to combine the acquired attenuation profile needed to reconstruct the image [18]. The automatic current modulation system of the CT must keep into account this relationship between pitch values, reconstruction algorithm and consequent noise and the observed results outlines the consequences on the patient dose. Radiologists should remind that with this CT equipment patient dose strongly increases when the pitch is reduced, even in the presence of the automatic tube current modulation system. A pitch value of 0.563 instead of a pitch of 0.938 causes a dose increase of $44 \%$ and also increases the noise, so the use of this pitch value should be justified by the need of other related improvements in image quality such as a better $Z$-axis resolution. Other CT equipments and tube current modulation systems showed a different relationship between the dose and the pitch factor: for example Papadakis et al. noticed a substantial constancy of the dose reduction for pitch factors in the range 0.5-1.25.

A limitation of our study is that our results may not apply to a different CT system and tube current modulation, but this is a common problem for the studies on this topic. We performed the measurements on another 16 slices GE equipment with a different $\mathrm{X}$-ray tube and generator, obtaining comparable results. It is reasonable to assume that the observed noise and dose trends are a characteristic of these equipments and of the "AutomA" software. As outlined in [19], although the use of CT automatic exposure control is generally quite straightforward, there are significant differences from one system to another. It is important that users are aware of the behaviour of their system, and the effect that varying scan and reconstruction parameters has upon the automatic exposure control.

Another limitation is that we perform our comparisons using an anthropomorphic adult phantom of medium size and we cannot extend these results directly to small or large size patient. Other studies [10] investigated the tube current modulation for pediatric patients of different sizes, but to our knowledge there are not adult anthropomorphic phantoms of different sizes commercially available and as a consequence it is only possible to make a clinical study to investigate this topic. A final limitation is related to the fact that other scan parameters, such as the beam width and the relative combination of detectors used and reconstructed slice thicknesses, may influence the current modulation, but in this study we decided to focus our attention to the tube voltage and the pitch factor maintaining constant the slice thickness used for this kind of examinations.
In conclusion, this study outlines the relationships between an automatic tube current modulation system and other CT acquisition parameters by mean of a characterization performed with an anthropomorphic phantom. The obtained results show that the NI can be used to obtain user-specified image noise also at different $\mathrm{kV}$ and pitch values, considering the consequences on the patient effective dose. We believe that these findings can be useful for the choice requested by radiologists in the task of optimization of the dose in CT acquisition protocols.

\section{Acknowledgements}

The study was supported, in part, by grants from the project "Riduzione del rischio associato all'esposizione a radiazioni ionizzanti per fini medici - Compagnia San Paolo di Torino".

\section{References}

[1] Kalra MK, Maher MM, Toth TL, et al. Techniques and applications of automatic tube current modulation for CT. Radiology 2004;233(December (3)):64957.

[2] McCollough $\mathrm{CH}$, Bruesewitz MR, Kofler Jr JM. CT dose reduction and dose management tools: overview of available options. Radiographics 2006;26(March-April (2)):503-12.

[3] Kalender WA, Wolf H, Suess C, Gies M, Greess H, Bautz WA. Dose reduction in CT by on-line tube current control: principles and validation on phantoms and cadavers. Eur Radiol 1999;9(2):323-8.

[4] Greess H, Wolf H, Baum U, et al. Dose reduction in computed tomography by attenuation-based on-line modulation of tube current: evaluation of six anatomical regions. Eur Radiol 2000;10(2):391-4.

[5] Tack D, De Maertelaer V, Gevenois PA. Dose reduction in multidetector CT using attenuation-based online tube current modulation. AJR Am J Roentgenol 2003;181(August (2)):331-4.

[6] Mulkens TH, Bellinck P, Baeyaert M, et al. Use of an automatic exposure control mechanism for dose optimization in multi-detector row CT examinations: clinical evaluation. Radiology 2005;237(October (1)):213-23.

[7] Kalra MK, Rizzo S, Maher MM, et al. Chest CT performed with $z$-axis modulation: scanning protocol and radiation dose. Radiology 2005;237(October (1)):303-8.

[8] Funama Y, Awai K, Nakayama Y, et al. Radiation dose reduction without degradation of low-contrast detectability at abdominal multisection CT with a low-tube voltage technique: phantom study. Radiology 2005;237(December (3)):905-10.

[9] Goo HW, Suh DS. The influences of tube voltage and scan direction on combined tube current modulation: a phantom study. Pediatr Radiol 2006;36(August (8)):833-40.

[10] Papadakis A, Perisinakis K, Damilakis G. Angular on-line tube current modulation in multidetector CT examinations of children and adults: the influence of different scanning parameters on dose reduction. Med Phys 2007;34(July (7)):2864-74.

[11] Kanal KM, Stewart BK, Kolokythas O, Shuman WP. Impact of operator-selected image noise index and reconstruction slice thickness on patient radiation dose in 64-MDCT. AJR Am J Roentgenol 2007;189(July):219-25.

[12] http://www.impactscan.org.

[13] Nakayama Y, Awai K, Funama Y, et al. Abdominal CT with low tube voltage: preliminary observations about radiation dose, contrast enhancement, image quality, and noise. Radiology 2005;237(December (3)):945-51.

[14] Nakayama Y, Awai K, Funama Y, et al. Lower tube voltage reduces contrast material and radiation doses on 16-MDCT aortography. AJR Am J Roentgenol 2006;187(November (5)):W490-7.

[15] Waaijer A, Prokop M, Velthuis BK, Bakker CJ, de Kort GA, van Leeuwen MS Circle of Willis at CT angiography: dose reduction and image quality-reducing tube voltage and increasing tube current settings. Radiology 2007;242(March (3)):832-9.

[16] Sahani DV, Kalva SP, Hahn PF, Saini S. 16-MDCT angiography in living kidney donors at various tube potentials: impact on image quality and radiation dose. AJR Am J Roentgenol 2007;188(January (1)):115-20.

[17] Sigal-Cinqualbre AB, Hennequin R, Abada HT, Chen X, Paul JF. Low-kilovoltage multi-detector row chest $\mathrm{CT}$ in adults: feasibility and effect on image quality and iodine dose. Radiology 2004;231:169-74.

[18] Hsieh J. Analytical models for multi-slice helical CT performance parameters. Med Phys 2003;30(February (2)):169-78.

[19] Nicolas Keat. CT scanner automatic exposure control systems. Report 05016 IMPACT. 